Revista

\title{
Multi-Ensayos
}

Vol. 7, núm. 13

ISSN: 2412-3285

https://multiensayos.unan.edu.ni

DOI: https://doi.org/10.5377/multiensayos.v7i13.10750

\section{El dirigente universitario ante el reto de la calidad educativa en el contexto de la crisis sanitaria COVID-19}

\section{The university leader faced the challenge of educational quality in the context of the COVID-19 health crisis}

\author{
Emilio Martín Lanuza Saavedra ${ }^{1}$
}

\section{RESUMEN}

Recibido: 09 de noviembre de 2020. Aceptado: 16 de noviembre de 2020

En este artículo se expone el papel del dirigente universitario en tiempos de emergencia, en el actual contexto como es el caso de la pandemia COVID 19, desde la perspectiva de la Universidad Nacional Autónoma de Nicaragua UNAN - Managua. Tener un cargo de dirección representa un reto, porque significa gerenciar la institución desde una perspectiva dinámica, cambiante e integradora de la política educativa de la institución que conlleve a alcanzar los objetivos institucionales, independientemente del nivel de dirección en la que actúe y de las circunstancias que el contexto va determinando. Actualmente, para hacerle frente al desafío de dirigir una entidad educativa, es esencial el uso de la tecnología, la que facilita la comunicación entre los involucrados, disminuye el tiempo en la realización de tareas optimizando recursos, a su vez, es una herramienta básica para que docentes realicen sus actividades de aprendizaje, desde una perspectiva dinámica, creativa e innovadora a la que los estudiantes responden con entusiasmo y aceptación por ser nativos digitales, no obstante, la tecnología no lo es todo, sin duda, facilita el desarrollo de procesos, pero en primera instancia está la relación docente - estudiante y estudiante - estudiante. Finalmente, los dirigentes deberán estar preparados previendo posibles situaciones de emergencia como la actual pandemia del coronavirus, para hacerle frente con determinación, madurez y sensatez a la crisis, disponiendo de todos los recursos: humanos, financieros, materiales y tecnológicos ejecutando acciones pertinentes para alcanzar la meta propuesta que es la formación integral de los estudiantes garantizando la calidad educativa aun en medio de las crisis.

Palabras clave: autoridades; universidad; educación de calidad; coronavirus; crisis.

\begin{abstract}
This article exposes the role of the university leader in times of emergency such as the COVID -19 pandemic such as at the National Autonomous University of Nicaragua UNAN - Managua. Having a management position represents a challenge because it means directing from a changing and integrating perspective of the educational policy of the institution that leads to achieving its objectives, regardless of the level of management in which it acts and the circumstances of the context. Currently, to face the challenge of running an educational entity, the use of technology which facilitates communication between those involved and reduces the time in carrying out tasks optimizing resources is essential. It is a basic tool for teachers to carry out their learning activities from a dynamic, 1 Docente Universidad Nacional Autónoma de Nicaragua. Doctor en Gestión y Calidad de la Educación. Correo electrónico: emilio.lanuza@gmail.com

(c) 2021 Revista Multi-Ensayos.
\end{abstract}


creative and innovative perspective to which digitally native students respond with enthusiasm and acceptance. However, although technology certainly facilitates the development of processes, of paramount importance is the teacher-student and student-student relations. Finally, the leaders must be prepared by anticipating possible emergency situations such as the current coronavirus pandemic and by facing the crisis with determination, maturity and good sense. It is necessary to have all the human, financial, material and technological resources to execute relevant actions to achieve the proposed goal of the comprehensive training of students, guaranteeing educational quality even in the midst of crises.Keywords: virtual learning environments; role of the teacher; role of the student; technological tools.

Keywords: authorities; university; quality education; coronavirus; crisis.

\section{INTRODUCCIÓN}

Actualmente las instituciones educativas están expuestas a situaciones complejas de administración y dirección, desde el manejo de personal, definición de líneas estratégicas, modelo educativo a implementar, dotación de tecnología, infraestructura, condiciones de trabajo, entre otras, que se agudizan con emergencias externas como el actual coronavirus, que indudablemente influye en los procesos que se realizan en estas instancias y que los dirigentes tienen que prever para tomar las decisiones acertadas y alcanzar lo propuesto institucionalmente.

Por su parte, los cambios vertiginosos que se viven en la sociedad de la información y del conocimiento, y el avance tecnológico, inciden en todos los ámbitos de nuestras vidas, nos han cambiado las formas de relacionarnos, hacer negocios, informarnos, diagnosticar, planificar, educarnos. La tecnología con el avance actual sirve como herramienta para facilitar el éxito o el fracaso de lo planificado dependiendo como se gestione e implemente para alcanzar las metas planificadas.

Por lo anterior, las personas que están a cargo de dirigir entidades educativas, se encuentran con el reto de visionar estratégicamente el quehacer de la institución, para integrar de forma armónica todos los recursos con los que cuenta la institución, en función de alcanzar los objetivos propuestos de la institución.

El dirigente siempre debe estar claro de la misión y visión institucional para darle cumplimiento a las planificaciones estratégicas, que conllevan a alcanzar los objetivos propuestos con la calidad requerida en cada una de las acciones que se realicen y más aún trasmitir a los colaboradores hacia donde se enrumba la institución y mantener la competitividad en los diferentes espacios educativos.

Por tanto, es todo un desafío lograr una dirección en la que los miembros de la institución estén claros y consientes de la responsabilidad que recae en ellos para alcanzar lo que pretende la institución, no cabe duda que mantener la armonía y equilibrio laboral depende de cada persona, pero también de la forma de dirigir de los tomadores de decisiones.

Por otro lado, estar al frente de una institución en tiempos de crisis, como la que se está viviendo actualmente con el COVID 19, complejiza la situación, involucra otros factores que el dirigente debe tomar en cuenta y que debe gestionar adecuadamente para mantener la estabilidad, control y orden laboral, 
por ejemplo: la parte emocional de las personas que conlleva temores, incertidumbres, miedos, otros. Los que se deben gestionar adecuadamente, desde la perspectiva individual y grupal para mantener el equilibrio emocional entre los que interactúan en la institución, parte importante para desarrollar con éxitos las actividades académicas, que al fin y al cabo es de lo que se ocupa la entidad.

\section{MATERIALES Y MÉTODOS}

Para la elaboración de este artículo se utilizó la metodología bibliográfica, partiendo de artículos científicos, ponencias, informes, folletos entre otros, así mismo se expresa la experiencia de dirección del autor, las vivencias y la toma de decisiones que se realizaron durante los meses más difíciles de la pandemia.

Parte de aspectos teóricos que en el ámbito de dirección se deben de tomar en cuenta, tales como: inteligencia emocional, uso de la tecnología, dirección horizontal, liderazgo, trabajo en equipo, trabajo colaborativo, lo que de forma armónica se conjuga con la experiencia de las autoridades para resolver situaciones de crisis y sacar adelante la institución cumpliendo con la planificación estratégica.

El método utilizado es el analítico, puesto que se parte de un análisis minucioso de los tipos de dirección de carácter administrativo, y se van destacando las características de estos, al relacionarlas con la práctica directiva que se implementan en la universidad. Además, se dan aportes de los elementos que un dirigente debe tomar en cuenta para tener éxito en la dirección de la institución, relacionando la teoría con la práctica misma, desde una concepción holística, integradora, que genere sinergia tanto a lo interno como a lo externo en el ámbito educativo.

\section{DESARROLLO}

Ser dirigente universitario implica una gran responsabilidad, porque se trata no solo de enfrentar "los nuevos paradigmas educativos" (Linares, Angulo y Villalobos, 2004, p. 299), debe tomar en cuenta las condiciones de infraestructura, personal y contexto en el que se encuentra la institución, así como del avance científico y tecnológico para dar respuestas pertinentes a las necesidades y demandas que el desarrollo educativo en particular y social en general exigen de una entidad educativa de orden superior.

La dirección de una entidad educativa universitaria se debe ver desde una perspectiva holística, en la que el motor que impulsa la institución se sustente en los ejes fundamentales, como son: la docencia, la investigación, la extensión y podríamos agregar un cuarto eje como es lo axiológico, es decir, los valores.

Sobre el dirigente, recae la responsabilidad de planificar y dirigir actividades con visión estratégica que favorezcan el desarrollo de la institución que conduce, con el objetivo de alcanzar las metas propuestas con la mayor calidad y obtener un producto por excelencia, "formación integral y de calidad de los estudiantes", que sean agentes de cambio en beneficio del desarrollo personal, comunitario y de país, adaptándose a las nuevas exigencias que demanda la sociedad en desarrollo (Linares, Angulo y Villalobos, 2004). 
Los dirigentes deben garantizar la ejecución del currículo con calidad, velar por el cumplimiento del Modelo Educativo adoptado, ir en busca de la misión y visión que la institución se ha definido lograr, en busca siempre del éxito y la calidad desde las diferentes instancias de dirección. Más aún, desde aquellas que están administrando el currículo de muy cerca como las direcciones de departamento y coordinadores de carrera, para garantizar la calidad educativa en función de la formación integral de los educandos.

Por lo anteriormente señalado, un dirigente educativo debe "incorporar elementos participativos orientados a mejorar la enseñanza y replicar las buenas prácticas pedagógicas" (Freire y Miranda, 2014, p. 11), que surgen con la experiencia de cada docente dentro y fuera del aula de clase y en ese interactuar con los estudiantes, así como desde las reflexiones críticas de su propia práctica pedagógica. Lo anterior sirve de inspiración y de referencia para otros docentes, y el papel del dirigente es canalizar estas experiencias y compartirlas que sin lugar a dudas enriquecerán el quehacer educativo en beneficio de los sujetos directos del aprendizaje, es decir, docentes y estudiantes.

Un dirigente debe tener liderazgo, y guiar a su institución "en el establecimiento de direcciones claras para la búsqueda de oportunidades de desarrollo" (Freire y Miranda, 2014, p. 12), debe guiar a la mejora de la institución ¿Qué implica esto?, implica que al cambiar la vida de la institución se estaría incidiendo significativamente en el cambio de todos los que tengan relación con esta: dirigentes, docentes, estudiantes, personal administrativo. Sin embargo, ese liderazgo debe ser compartido para la toma de decisiones estratégicas, colegiado para el éxito de la institución.

Es importante considerar el elemento innovador en la dirección de la universidad, visto como una oportunidad para modificar elementos existentes con el fin de mejorarlos o bien considerar elementos nuevos que dinamicen el quehacer institucional de acuerdo al contexto, necesidades y demandas que contribuyan al crecimiento de la institución y al logro de los objetivos institucionales. Es reflexionar el proceso y prever los resultados anticipándose a los mismos, de tal manera que se puedan predecir para evitar resultados no deseados que irán en detrimento del logro de las metas propuestas.

En tiempo de crisis, se "requiere mantener simultáneamente una visión coherente a largo plazo y un conjunto de soluciones efectivas a corto plazo, atendiendo a los micro-procesos" (Montecinos, Uribe y Volante, 2020, p. 7), se debe actuar de forma clara y precisa, mostrar dominio de la situación y lo que se oriente esté acorde a los principios básicos de funcionamiento de la institución, es como enfocarse en alcanzar las metas que en esencia rigen a la institución educativa, "formación integral de los estudiantes".

Es importante que el dirigente escuche a su personal, indague sobre lo que piensan y sienten, conviene aprovechar a aquellas personas con mayor experiencia que propongan acciones pertinentes a realizar para que las decisiones que se tomen sean las más acertadas.

Los líderes institucionales deben tener clara la visión y misión de la institución, con ello estar conscientes de la razón de ser, el propósito y las aspiraciones que la institución se propone alcanzar en el corto, mediano y largo plazo, deben empujar hacia el logro del Modelo Educativo. En el caso de la UNAN Managua, "está centrado en la persona y su formación integral, con una concepción científica y humanista, 
capaces de interpretar los fenómenos sociales y naturales con un sentido crítico, reflexivo y propositivo" (UNAN Managua, 2011, p. 7). Al tener claro lo anterior, el dirigente podrá dinamizar la institución, perfilar las estrategias institucionales, adecuarlas y adaptarlas a las diferentes situaciones que se presenten de acuerdo al contexto educativo y a las necesidades que surgen en la sociedad.

De ahí que, en situaciones como la pandemia, la crisis se convierta en una oportunidad de aprendizaje, en toda una escuela que nos permita enfrentar el fenómeno con iniciativa creadora y con estrategias innovadoras para mantener la calidad de la educación.

Desde la experiencia de la Universidad Nacional Autónoma de Nicaragua y en particular de la Facultad Regional Multidisciplinaria Estelí, se realizaron cambios en cuanto al desarrollo del primer semestre académico 2020, para dar respuesta a la continuación del mismo. Se pasó de una actividad regular a la metodología por encuentro, asimismo se realizó división de grupos para mantener el distanciamiento y la protección de docentes y estudiantes.

Se incluyó un horario reducido en el que la jornada laboral para todos los colaboradores de la institución iniciaba a las 08:00 a.m. y finalizaba a la 01:00 p.m. Por otra parte, los trabajadores con mayor riesgo ante el contagio por el coronavirus, tenían un horario especial para hacer presencia en la Facultad, y la mayor parte de su jornada la cumplían desde sus hogares con una conexión en línea, haciendo uso de la tecnología.

En la parte académica, se realizaron ajustes a los programas, a partir de una selección de contenidos para las clases presenciales y apoyándose de la tecnología para dar seguimiento a los procesos de aprendizaje de los estudiantes. Todo con el fin de garantizar que todos los estudiantes, incluso los que no asistían a los encuentros presenciales por diferentes razones pudieran continuar con sus estudios y culminar con el semestre exitosamente.

Desde la parte de la infraestructura, se instalaron lavamanos en todos los edificios, para proporcionar los requerimientos para el lavado de manos, control de temperatura a la entrada para todas las personas que ingresaban a las instalaciones, así como el rociado de manos con alcohol y el uso exigido de la mascarilla. Además, se realizaron charlas de sensibilización en cuanto a la importancia de mantener las medidas higiénicas sanitarias para prevenir el contagio por el virus SARS-CoV-2.

Lo anterior se considera de un acierto por parte de las autoridades de la Facultad, así también de las autoridades del nivel central que autorizaron la realización de ajustes necesarios para garantizar la continuidad del semestre y a su vez, tomar las medidas de seguridad y protección para la continuidad del semestre.

Un aspecto importante en todo esto, ha sido el uso de la tecnología, en todas las divisiones administrativas y académicas, que han permitido la realización de las actividades y la continuidad del trabajo. Sin la tecnología, impulsar cambios acordes a las demandas educativas actuales y hacerle frente a la emergencia que se presentan tanto interna como externa, implicaría serías limitaciones, sin embargo, la misma se 
debe usar racionalmente y tener presente que, su utilidad no sustituye la relación socio-educativa del docente y el estudiante.

Por lo anterior, las Tecnologías de la Información y la Comunicación (TIC), deben ser ampliamente aprovechadas para la facilitación de procesos educativos y de aprendizaje, igualmente los Recursos Educativos Abiertos (REA) que contribuyen en gran medida en el aprendizaje y en el desarrollo intelectual de las personas, estos recursos deben ser bien aprovechados en las instituciones educativas.

Los dirigentes deben estar conscientes del potencial de estos recursos y herramientas que bien gestionadas producen grandes resultados en la calidad y mejora educativa de toda institución. Deberán estar actualizado en los avances tecnológicos, gestionar tecnología de punta para su institución y garantizar la formación del personal docente en el uso y manejo de la tecnología, con el único fin de ser implementada en los procesos académicos que contribuyan en la formación de los estudiantes.

Sin embargo, hoy tenemos que hablar en primer lugar de las Tecnologías del Aprendizaje y el Conocimiento (TAC). Que en el contexto educativo tratan de reconducir las TIC hacia un uso más formativo y pedagógico, enfocándose específicamente en el proceso de enseñanza - aprendizaje, adaptando las exigencias educativas actuales a la forma de vida cotidiana de los estudiantes con la tecnología.

Sin duda las TAC vienen a fortalecer el ejercicio docente, porque son un producto de los usos genuinos de las TIC, "con el propósito de aprender de una mejor forma, estableciendo dinámicas y prácticas formativas que implican exploración de las variantes usos didácticos de la tecnología digital" (Pinto, Díaz y Alfaro, 2016, p. 40). Y en la práctica de la Facultad, es lo que los docentes siempre están implementando para que los estudiantes alcancen el aprendizaje.

La tecnología nos acerca a la información, al conocimiento actualizado, a facilitarnos los procesos de planificación, a optimizar el tiempo y los recursos en fin a dinamizar y agilizar las actividades que se realizan en la institución, por tanto un adecuado uso de esta, conlleva a que la institución sea competente y con las credenciales necesarias para optar a ser una institución educativa acreditada a nivel internacional, lo que da un prestigio y credibilidad en primer instancia a la institución misma y en segundo lugar a los egresados de ella.

Sin embargo, esa acreditación, debe tener también el respaldo de docentes calificados, con producción científica, con el desarrollo de programas de estudio pertinentes y con metodologías que respondan a la formación de los educandos.

Con la implementación de las herramientas tecnológicas se pueden desarrollar procesos educativos virtuales, que refuercen los procesos educativos presenciales y proporcionen a los estudiantes y docentes espacios de comunicación e intercambio lo suficientemente adecuados. Lo anterior facilitará procesos de aprendizaje sólidos y pertinentes que garanticen la adquisición de las competencias requeridas en su carrera y que responda a las demandas de trabajo actuales. 
Además, con procesos educativos virtuales, se puede desarrollar un programa de asignatura, realizar actividades de aprendizaje y evaluaciones sin la necesidad de estar en un espacio físico presencial para realizar este proceso, la tecnología nos acerca tanto como lo queramos y también nos aleja de la misma forma, por tanto, debe ser bien utilizada.

A pesar de lo mencionado anteriormente, hay que tener cuidado con el uso de la tecnología, en las instituciones educativas se trabaja esencialmente con personas y las personas necesitan del acercamiento físico, la tecnología "puede modificar las relaciones humanas, generando un nuevo orden social" (Gordón, 2011, p. 155), esta puede distanciar a las personas del contacto físico y de las relaciones afectivas que son de importancia para generar un ambiente adecuado de trabajo y facilitar procesos de intercambio y aprendizaje. Por eso en la Facultad, la experiencia que hemos tenido al relacionar lo virtual con lo presencial, siempre ha estado presente la relación humana, como un elemento primordial de humanización de la praxis educativa.

Se debe considerar que en toda institución educativa se establecen múltiples relaciones, entre las principales podemos destacar la relación docente-docente, docente-estudiante, estudiante-estudiante, dirigentes - colaboradores, entre otras. Esta última, es clave en el bien andar de la institución, un equipo en el que se sienta en confianza con su superior, que les trasmita seguridad y escuche las propuestas del grupo de trabajo, es un equipo fuerte y cohesionado, que trabaja por la institución y porque todas las actividades que se realizan salgan bien.

Por lo anterior, un dirigente debe ser "democrático, participativo, descentralizador en la toma de decisiones, posibilitador, animador y comprometido con el cambio" (Peniche Cetzal, Ramón Mac, Rosario Muñoz, 2018, p. 89), aún más si realiza su trabajo de forma "extra - ordinaria, al salirse de lo habitual" (Moreno, 2012, p. 4). Manteniendo el contacto permanente con sus colaboradores, conversando con ellos y escuchándolos, esta actitud lo llevará a realizar los cambios que considere contando con el apoyo decidido y comprometido de todos los que laboran en la institución. Esa ha sido la experiencia de la Facultad en la actual crisis sanitaria, las diferentes instancias de dirección han sabido conducir el proceso pedagógico y administrativo de la educación.

Al conducir la institución desde este punto de vista, entonces se está hablando del trabajo colaborativo y complementario que todas y todos deben realizar para sacarla adelante, recordar que es lo más importante. De esta forma se crea la identidad institucional y todos son un solo equipo.

Las autoridades deben tener presente que un equipo de trabajo colaborativo es "un conjunto o grupo de personas que poseen capacidades complementarias y que colaboran para alcanzar unos objetivos comunes compartiendo responsabilidades" (Jaramillo, 2012, p. 7), desde este punto de vista, el dirigente debe en primer lugar confiar en su equipo de trabajo, asignar responsabilidades y monitorear el cumplimiento de las mismas, sin que los miembros del equipo se sientan presionados o fiscalizados en el cumplimiento de la tarea, sino más bien que se establezca una comunicación en la que se puedan ver aquellos detalles que garantizarán el éxito de la actividad. 
Otro aspecto que las autoridades deben considerar al trabajar con personas, son las emociones y sentimientos de su personal, que influyen considerablemente en el rendimiento productivo del trabajo, como ya se ha mencionado anteriormente, así como en las relaciones bilaterales que se establecen entre cada uno de los miembros del equipo y la relación con el dirigente.

Se debe cuidar que el equipo se sienta bien emocionalmente con lo que hace y con las relaciones interpersonales que se establecen, debe prevalecer el respeto y el consenso para mantener la armonía del trabajo y el equilibrio emocional de cada uno. El estado emocional se debe trasmitir a los estudiantes, porque al final, ellos son la razón de ser de la institución. Recordemos que hoy en día, gran parte del éxito de los procesos educativos, se encuentra en este aspecto.

Es importante tener en cuenta que, la razón y la emoción para la toma de decisiones juegan un papel fundamental, un dirigente que mantenga un equilibrio entre ambas tiene mayores posibilidades de éxito en las decisiones que tome, en cambio, si nos llevamos por una de ellas existen más posibilidades de fracaso.

La razón y la emoción:

son dos formas fundamentales de conocimiento que interactúan para construir nuestra vida mental [...] y en todo momento se debe buscar su equilibrio, ya que los sentimientos son indispensables para la toma racional de decisiones, porque nos orientan en la dirección adecuada para sacar el mejor provecho a las posibilidades que nos ofrece la fría lógica. (Ibarrola, 2009, pp. 1-2)

Lo anterior refiere que una persona estable emocionalmente, actuará de forma racional previendo conscientemente las ventajas y desventajas ante una situación concreta, y las decisiones que tome serán acertadas, por ende, tanto el líder como sus colaboradores deberán estar equilibrados emocionalmente.

Ante una emergencia como la crisis que ha provocado el coronavirus, las autoridades deben de actuar con calma y serenidad, dominando la inteligencia emocional para gestionar adecuadamente las relaciones que se establecen con sus colaboradores y enrumbar el trabajo en la institución hacia la estabilidad en medio de la crisis, y sobre todo generando seguridad y estabilidad en los estudiantes.

Toda crisis genera cambios, nos hace reflexionar sobre lo que hacemos y cómo lo hacemos, nos lleva al punto de hacer un alto en el camino y revisar lo que hemos venido haciendo y analizar los resultados obtenidos, por ende, nos lleva a un proceso de aprendizaje y de crecimiento personal e institucional.

Por lo anterior, enfrentar la crisis desde el papel de las autoridades universitarias, no es una tarea sencilla o fácil, es compleja, es retomar lo que se ha expuesto en este artículo relacionado con el papel del dirigente y sobre todo ponerlo en práctica, es ver las ventajas y las posibilidades que la institución tiene para continuar con el funcionamiento sin perder la calidad, mantener el equilibrio laboral de los colaboradores para el buen andar de la institución. Además de conducir y potenciar lógicamente el trabajo educativo con los estudiantes. 
Hay que recordar que "la emoción es más fuerte que el pensamiento, incluso puede llegar a anularlo" (Ibarrola, 2009, p. 2), prestar atención a las manifestaciones emocionales de las personas que laboran en una institución es fundamental y clave y más aún en tiempo de emergencia como la actual.

En conclusión, un líder en tiempos de crisis debe mostrarse empático, humano y auténtico; debe ser realista en el análisis y en los problemas que se plantean, pero sin transmitir negatividad; y debe potenciar la comunicación y el buen ambiente de trabajo creando un clima de confianza y seguridad. Precisamente, eso es lo que ha pasado en nuestra Facultad en el marco de esta crisis sanitaria, los dirigentes, han estado a la altura de las circunstancias y eso ha permitido dar saltos de calidad con el esfuerzo de toda la comunidad universitaria.

\section{CONCLUSIONES}

Las autoridades universitarias bajo esta emergencia sanitaria tienen un reto, y es mantener la calidad educativa en la institución, siendo conscientes que primero es el bienestar de las personas con las que se interactúa en la institución, docentes, estudiantes, trabajadores administrativos, comunidad universitaria.

La tecnología juega un papel esencial en el apoyo de las actividades educativas, con esta se mantiene la comunicación fluida entre autoridades, docentes, estudiantes, con ella se facilita la información en todos los niveles y lo más importante aún que facilita las actividades de aprendizaje, de intercambio y se puede dar seguimiento al desarrollo de las expresiones educativas que se impulsan desde las diferentes instancias académicas.

El trabajo en la institución se debe organizar de tal manera que sea colaborativo, formar equipos de trabajo en el que el dirigente se deberá involucrar y estar al frente para tomar las decisiones que se susciten y requieran de una respuesta inmediata y acertada, que permita a la autoridad tener una dirección de proceso para elevar los resultados de la institución hacia niveles de calidad propuestos.

La estabilidad emocional debe partir de las autoridades, estos deben mostrarse y actuar de forma equilibrada e irradiar seguridad a los demás miembros de la institución y en todo momento mostrar seguridad ante las decisiones que se vayan ejecutando. Teniendo en cuenta el análisis y la evaluación del resultado que se vaya obteniendo de estas, que permitan la realización de ajustes pertinentes para alcanzar los mejores resultados.

Es conveniente ver la crisis o emergencia como una oportunidad para aprender, renovar y crecer y que las lecciones que deja sirvan para dinamizar la institución con el fin siempre de mejorar cada uno de los recursos que dispone la entidad.

Por el último, el dirigente deberá estar abierto a los cambios, enfrentar sus temores y miedos para tomar decisiones oportunas, adecuadas a la situación planteada, y si una decisión no sale como se esperaba, tener la capacidad de realizar el cambio oportuno en busca del mejor resultado. 


\section{REFERENCIAS}

Freire, S., y Miranda, A. (2014). El rol del director en la escuela: el liderazgo pedagógico y su incidencia sobre el rendimiento académico (Vol. 17). https://bit.ly/3jiSH3o

Gavotto, O. I., Glasserman, L., Monge, P., y Castellanos, L. (2015). La Cohesión del Colectivo, como Factor Clave para el Trabajo Colaborativo en Educación Básica. Revista Iberoamericana de Evaluación Educativa, 8(2), 171-185. https://bit.ly/3aXK7V3

Gordón, F. A. (2011). Refleciones filosóficas sobre la Tecnología y sus nuevos escenarios. Dialnet, 123-174. https://bit.ly/3InUJ48

Ibarrola, B. (2009). Dirigir y educar con Inteligencia Emocional. Ponencia del VII congreso de Educación y Gestión, 1-10. https://bit.ly/3gBQmid

Jaramillo, R. (2012). Trabajo en equipo. https://bit.ly/31sZIZw

Lanuza, E. (2019). Tecnologías de la Información y Comunicación integradas en estrategias didácticas innovadoras que faciliten procesos de enseñanza aprendizaje en la unidad de funciones de Matemática General, FAREM Estelí.

Linares, J., Angulo, E., y Villalobos, F. (2004). Funciones directivas y perfil de competencias del gerente educativo en instituciones de educación básica. Telos: Revista de Estudios Interdisciplinarios en Ciencias Sociales, 6(2), 298-318. https://bit.ly/32sv023

Montecinos, C., Uribe, M., y Volante, P. (2020). Liderazgo Escolar: Aprendiendo en tiempos de crisis. Santiago de Chile. https://bit.ly/3b6zK15

Moreno, C. (2012). Liderazgo humanista y acción directiva. Red Tercer Milenio S.C., 11. https://bit. ly/2Qs2UOH

Peniche Cetzal, R. S., Ramón Mac, C. C., y Rosario Muñoz, V. M. (2018). El liderazgo escolar entendido desde el directivo y el profesorado en educación superior. Revista Internacional de Organizaciones, (21), 85-108. https://doi.org/10.17345/rio21.85-108

Pinto Santos, A. Díaz Carreño, J. y Alfaro Carmargo, C. (2016). Modelo Espiral de Competencias Docentes TICTACTEP aplicado al Desarrollo de Competencias Digitales. Revista Educativa Hekademos, (19), 39 - 48. Recuperado de https://dialnet.unirioja.es/servlet/articulo?codigo $=6280715$

Torres-Barzabal, L., y Morón-Marchena, J. (2017). Innovación docente Experiencias universitarias en Educación Social. https://bit.ly/3gxxQrB

UNAN-Managua (2011). Modelo Educativo, Normativa y Metodología para la Planificación Curricular. http://bit.ly/2KqiwzE 